\title{
HEAT TRANSFER AND FLUID FLOW INSIDE DUCT WITH USING FAN-SHAPE RIBS
}

\section{Ahmed Hashim Yousif}

Al-Furat Al-Awsat Technical

University, Al-Dewaniyah

Technical Institute,

Al-Dewaniyah, Iraq

Key words: heat transfer, fluid flow, fan-shape ribs, Nusselt number, CFD

\section{Cite article:}

Ahmed, H. Y. [2021]. Heat transfer and fluid flow inside duct with using fan-shape ribs. Journal of Applied Engineering Science, 19(2), 526 - 532. DOI:10.5937/jaes0-28398

Online aceess of full paper is available at: www.engineeringscience.rs/browse-issues 


\title{
HEAT TRANSFER AND FLUID FLOW INSIDE DUCT WITH USING FAN-SHAPE RIBS
}

\author{
Ahmed Hashim Yousif* \\ Al-Furat Al-Awsat Technical University, Al-Dewaniyah Technical Institute, Al-Dewaniyah, Iraq
}

A 2-D computational analysis is carried out to calculate heat transfer and friction factor for laminar flow through rectangular duct with using fan-shape ribs as a turbulator. The types of rib shapes are imported on the heat transfer rate and fluid flow in heat exchangers. The present study makes use of a fan-shaped ribs with two arrangements. The first arrangement was downstream fan-shape ribs (case 1) and upstream fan-shape ribs (case 2) is investigated. A commercial finite volume package ANSYS FLUENT 16.1 are used for solving the meshing process with continuity, momentum and energy equations respectively to investigate fluid flow and heat transfer across the ribs surface. The Reynolds number $(R e)$ range of $(400-2250)$ with different relative roughness pitch $(p / H=0.17,0.22,0.27$ and $0.32)$ at constant rib high $(\mathrm{e} / \mathrm{H})$. The results show that the heat transfers and friction increase with using ribs also, the results show that heat transfer is directly proportional to pitch ratio and Reynolds number. The Nusselt number enhancement by $(12 \%-29 \%)$.

Key words: heat transfer, fluid flow, fan-shape ribs, Nusselt number, CFD

\section{INTRODUCTION}

Heat transfer is increasing by using swirl. The swirl of fluid flow devices can be divided into two types. The first one is a twisted tape that lead to continuous swirl along the duct, the second device makes the flow decays along the channel is a ribs or baffle and vortex generator which is where the ribs success fully prevents the development of B.L. and lead to improving heat transfer. Various types of ribs have been developed to increasing heat transfer from rectangular duct where the rectangular duct is commonly used in engineering applications. Such as solar collector, compact heat exchanger etc. [1]. Enhancement of heat transfer is very importance in the thermal system where overheated can damage the components of the system [2]. The mechanism of enhancement heat transfers by using ribs is owing to the flow separation and reattachment where the laminar sub layer was disrupting with using different types of ribs. The ribs turbulator. Investigates recreation of B.L which lead to enhancement heat transfer [3]. Several of researchers have been studied on improvement heat transfer with using different types of ribs.

[1] Investigate heat transfer and friction factor for turbulent flow inside duct with triangular ribs numerically it was found that the ribs will be increases Nusselt number and pressure drop. [2] Studied a thermal performance characterization in duct with using different type of ribs at constant heat flux numerically. The $\mathrm{k}-\varepsilon$ model is used to simulate turbulence in ANSYS-FLUENT 6.3. Heat transfer enhancement with using ribs when compared with smooth duct and found that the highest performance factors for turbulent flow.

[3] Presented the numerical results of heat transfer and friction factor for turbulent flow through a square channel with using different shape of ribs with Re range (5000-
$52000)$ and different ratio (p/e). The increment in Nusselt number with using square ribs is 1.19 and friction factor lowered by 1.3 as compared with other type of ribs. [4] studied heat transfer and friction in air solar collector with using rectangular ribs numerically the results show that heat transfer enhanced without big penalty friction losses. [5] A different shaped of ribs at the same height with constant heat flux are using (fig. 1) the ribs using with two types of arrangement (in line and staggered) with Re range of (4000 to 12000), heat transfer and friction losses increasing with using ribs when compared with smooth channel, the right angle triangle give max. friction loss and reverse pentagonal shape give max. Nu.

[6] Used three shapes of ribs to investigate heat transfer of two phases flow, with three air velocities and three water velocities, heat transfer increases with adding ribs, by $73.97 \%$ for rectangular rib, with $99.14 \%$ for trapezoidal rib and with $135.65 \%$ for triangular rib when compared it's with smooth duct. [7] A numerical investigation on ribbed duct with T.T insert and compared with T.T insert

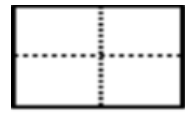

Case 1

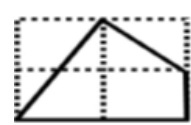

Case 5

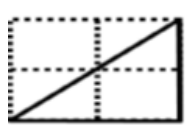

Case 9

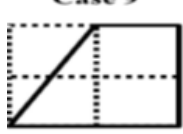

Case 13

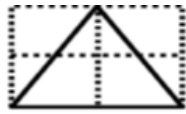

Case 2

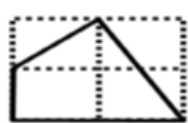

Case 6

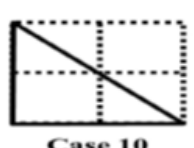

Case 10

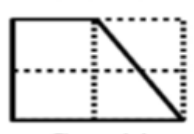

Case 14

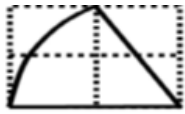

Case 3

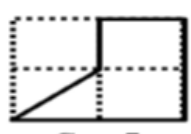

Case 7

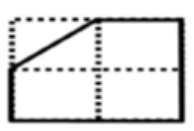

Case 11

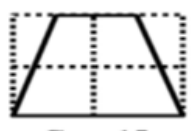

Case 15

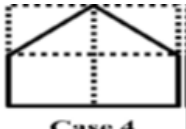

Case 4

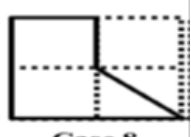

Case 8
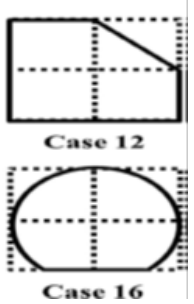

Case 16
Figure 1: Cross-Sectional rib shapes [5] 
in duct without using rib. The result shows that using ribs with T.T. lead to increasing heat transfer, also shows that higher twist ratio with moderate rib spacing with lower twist ratio for low Prandtl fluid.

[8] An orifical rib on plate to improving heat transfer from solar air heater was investigated numerically with $\mathrm{Re}$ range from 3000 to 20000 . The ratio of $(p / e=10)$ has the better results of heat transfer and the ratio of roughness height and pitch has very strong effect on the flow pattern. The study using five types of ribs and right angle triangle shape has higher performance. [9] Using of a semi-D shaped turbulator as a novel rib is using to enhancement heat transfer from flat plate solar air heater numerically at $\mathrm{Re}$ range (6000-27000) with $\mathrm{p}$ ratio (p/e) varied between (7.14-42.86). The results show that the heat transfer enhanced with maximum increasing in $\mathrm{Nu}$ about 2.43 at $p / e=7.14$ at $R e=6000$ with increasing in friction factor.

[10] studied the effect of rectangular ribs over observer plat for solar air collector at constant heat flux with $\mathrm{Re}$ range (4000-18000) numerically. The predicted results show that maximum value of performance was 1.89[11] Have numerically studied heat transfer and flow through a duct with triangular ribs at different angles by ANSYS 14 ICEM, the Re range between (20000 to 60000). The maximum heat transfer obtained for triangular ribs at angle $60^{\circ}$ and $\mathrm{Re}=60000$ when compared with angles $45^{\circ}$ and $90^{\circ}$ also, vortex created between the ribs improve Nu. An upstream and downstream ramped ribs are studding to show its effect on heat transfer and pressure drop. Experimentally and numerically by [12] the results compared with square ribs with the same height with turbulent flow at Re range (10000-60000). From this study, heat transfer and friction factor has a considerable penalty up to $28 \%$ and $43 \%$ respectively. However, the deficit in friction and heat transfer have significant impact on the air foil life.

[13] A computational analysis is carried out to show the effect of ten types of ribs on heat transfer and pressure drop using commercial package FLUENT 6.1 with Re range (3000-20000). The study is carried out for both flow regimes (transitional and fully develop). The result shows that optimum heat transfers with minimum pressure drop given with rectangular ribs. In addition, several researchers as [14-16] studies on using a variety of ribs and the results were that the heat transfer is improved with using ribs with increasing in the coefficient of friction. Several reviewed have been done by many researchers for the purpose of collecting researches and studies on the use of roughened surfaces to improve heat transfer as $[17,18]$.

In present study, the effect of fitting fan-shaped ribs in duct at constant wall temp. on heat transfer and pressure drop at Reynolds number range (400-2250) with upstream and downstream fan-shape ribs cases at different pitch ratio $(p / H)$ will be investigated numerically.

\section{SOLUTION DOMAIN}

The physical properties of numerical study are shown in fig. (2-a, b), the internal, test and exit sections can be shown and the dimensions of the duct exibited in table (1)

\section{CFD MYTHOLOGY}

The numerical calculation is performed to solve 2-D problem to know heat transfer and friction inside duct with using wing-shape ribs. A CFD cod FLUENT 6.1 is used to solve conservation equations. The eq.s are used to solution heat transfer and friction with the assumption of

- Steady two dimensional air flow and heat transfer

- Laminar incompressible with fully developed flow

- Constant air properties

- The thermal radiation, body force and viscose dissipation are ignoring.

The 2-D continuity, momentum and energy eq.s are solved with using F.V.M and SIMPLIC algorithm scheme is applied to show the effect of flow on heat transfer and fiction factor characteristic. A governing eq.s for present

Table 1: Dimensions of the duct and rib with respect to the height of duct

\begin{tabular}{|c|c|c|}
\hline Configuration & Sample & Dimensions value \\
\hline Pitch & $\mathrm{p} / \mathrm{H}$ & $\begin{array}{c}0.17,0.22, \\
0.27,0.32\end{array}$ \\
\hline Rib height & $\mathrm{e} / \mathrm{H}$ & 0.05 \\
\hline Entrance section & $\mathrm{L}_{1} / \mathrm{H}$ & 10 \\
\hline Test section & $\mathrm{L}_{2} / \mathrm{H}$ & 7.5 \\
\hline Exit section & $\mathrm{L}_{3} / \mathrm{H}$ & 12.5 \\
\hline Rib length & $\mathrm{b} / \mathrm{H}$ & 0.17 \\
\hline
\end{tabular}

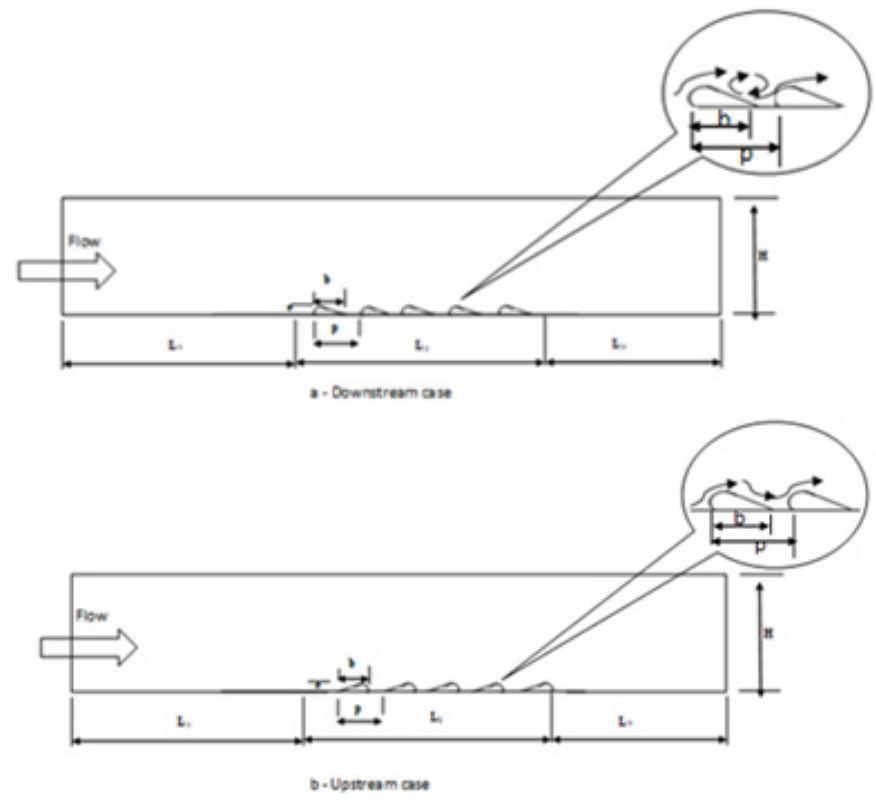

Figure 2: Downstream and upstream ribs 
study are written as [19, 20]:

Continuity equation

$\frac{\partial u}{\partial x}+\frac{\partial v}{\partial y}=0$

$\mathrm{x}$-momentum equation

$u \frac{\partial u}{\partial x}+v \frac{\partial v}{\partial y}=-\frac{\partial p}{\partial x}+\frac{1}{R e}\left(\frac{\partial^{2} u}{\partial x^{2}}+\frac{\partial^{2} u}{\partial y^{2}}\right)$

$\mathrm{y}$-momentum equation

$u \frac{\partial u}{\partial x}+v \frac{\partial v}{\partial y}=-\frac{\partial p}{\partial y}+\frac{1}{R e}\left(\frac{\partial^{2} u}{\partial x^{2}}+\frac{\partial^{2} u}{\partial y^{2}}\right)$

Energy equation:

$u \frac{\partial T}{\partial x}+v \frac{\partial T}{\partial y}=\frac{1}{\operatorname{Pr}}\left(\frac{\partial^{2} T}{\partial x^{2}}+\frac{\partial^{2} T}{\partial y^{2}}\right)$

\section{MESH GENERATION}

Mesh were implemented to the flow domain are shown in (fig.3) where the meth was very fine near the solid wall. It's necessary ensure the accuracy and validity of the results by checking the meth independence. Therefore, the meth independence study is done on the duct. The parameters for the present study are Nusselt number and friction factor. Convergence criteria for mass and momentum eq.s are $\left(10^{-6}\right)$ and for energy eq. is $\left(10^{-9}\right)$.

\section{CALCULATION OF NUSSELT NUMBER AND FRICTION FACTOR}

Nusselt number characterize the quantitative parameter of heat transfer where the form of Nu based on hydraulic diameter is:

$N u=\frac{h D_{H}}{k}$

And friction factor can be calculated by:

$f=\frac{\Delta P}{1 / 2 \rho U^{2}} \frac{D_{H}}{L}$

At solution of present study be at Reynolds number range (400-2250), where

$$
R e=\frac{\rho u D_{H}}{\mu}
$$

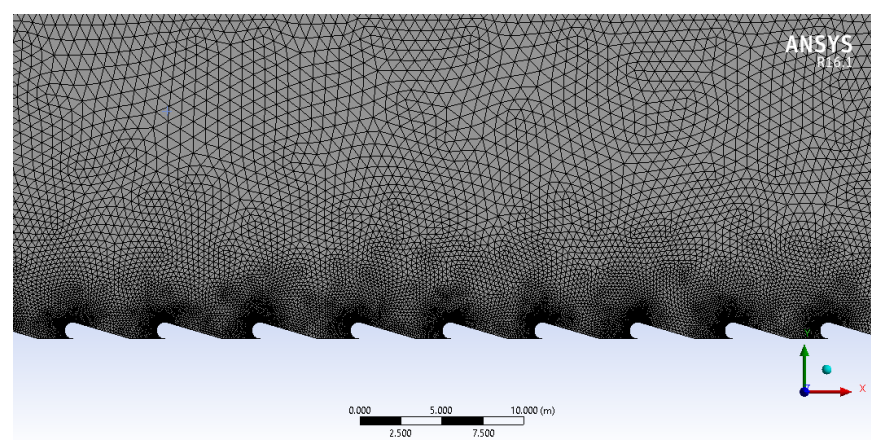

Figure 3: Mesh generation for downstream ribs

\section{Boundary conditions}

For this study, the pertinent (B.C) is the simulation are:

- Inlet boundary

u=uin

$\mathrm{v}=0$

Tin=300 K

- $\quad$ Solid boundary

No slip condition for velocity at walls (duct and ribs) with constant wall and ribs temperature of $300 \mathrm{k}$ and $400 \mathrm{k}$ respectively.

- Outlet boundary

The exit boundary condition are unknown before solving the eq.s and its principle of zero except pressure gradient sitting as

$\frac{\partial p}{\partial x}=0$

\section{RESULTS AND DISCUSSION}

First we must perform verification of heat transfer and friction for plain duct with previous studies. The verification is performed in term of Nu and $f$. the present numerical results are compared with [21] as shown in figure (4) for $\mathrm{Nu}$ and (5) for friction factor (f). the present numerical results are within $+10 \%$ of the results obtained from [21].

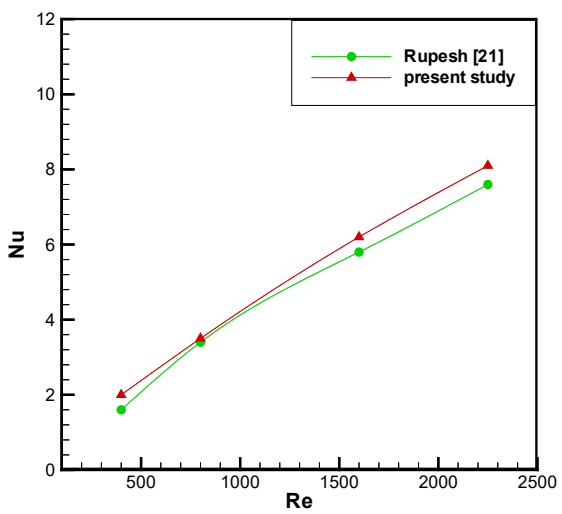

Figure 4: Nu with Re for plain duct

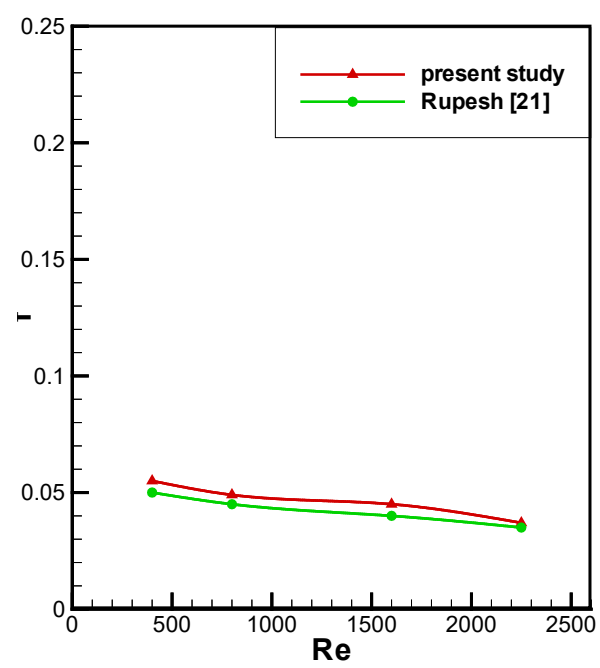

Figure 5: Friction factor with Re for plain duct 
CFD contour of temp. magnitude for downstream and upstream fan-shape ribs can be shown in figure (6) at different pitch ratio, where for downstream at small pitch ratio $(\mathrm{p} / \mathrm{H}=0.17)$ (fig.6-a), heat transfer enhanced at the tip of ribs facing the flow but when moving to the straight surface, heat transfer decreases due to generate vortices. But for upstream rib heat transfer was high at the straight surface due to sweeping the heat by the flow on the ribs (fig.6-e). Now for downstream ribs with increasing pitch ratio, the vortices will be disappearing and heat transfer will be increasing due to sweeping the heat from the ribs (fig.6-b, c, d). On the contrary, we show that vortices will be appearing and growth with increased pitch ratio where heat transfer decreases (fig. 6- f, g, h).

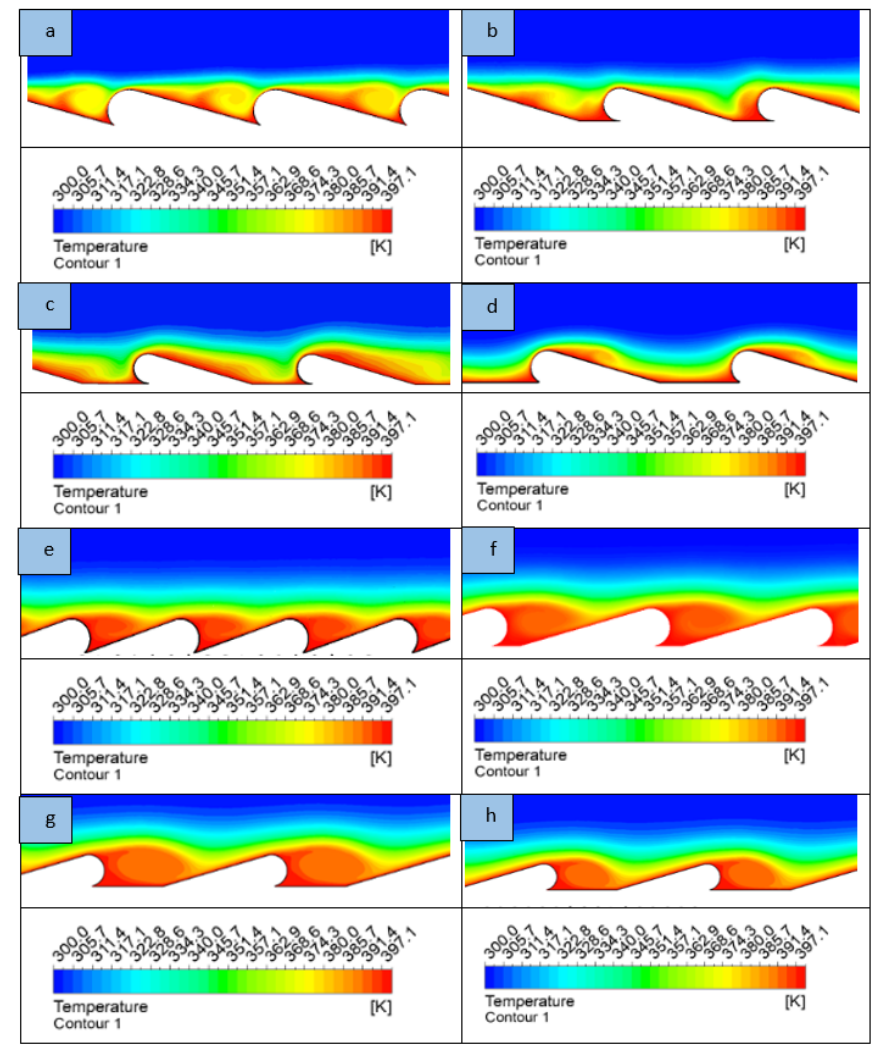

Figure 6: Temperature contour for downstream and upstream ribs
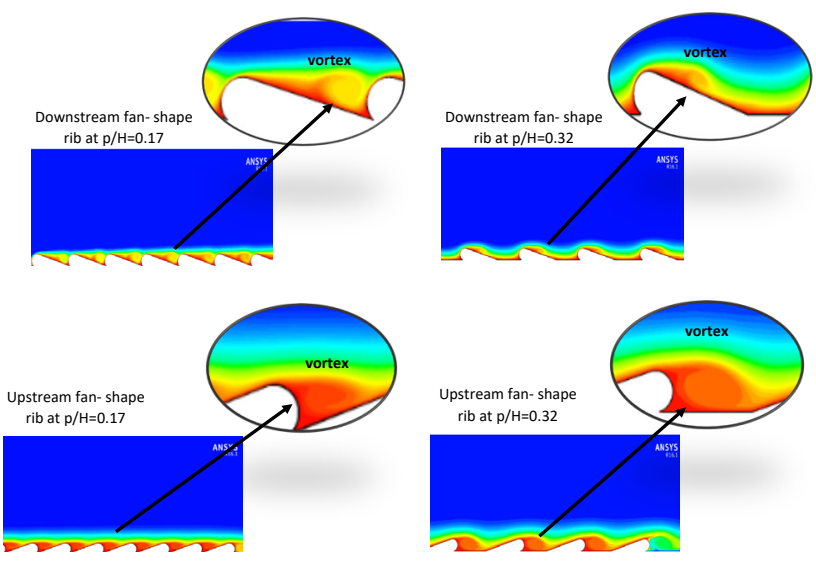

Figure 7: Vortices over ribs at different $(p / H)$ for two cases
(Fig.7) shows the effect of downstream upstream flow with different pitch ratio on generate vortices where it clear that the vortices depended on the flow direction and the pitch ratio.

(Fig.8 a-h) shows the velocity vectors for flow over the ribs. Where (fig.8-a) shew the velocity distribution at $(\mathrm{p} / \mathrm{H}=0.17)$, it is clear that the vortex will be appears also, there is a region recession at the end of straight surface of rib. But with increasing pitch ratio vortices will be decreasing and the flow be smooth over rib surface. In the case of upstream, at small pitch ratio (fig.8-e), there is a small vortex be at the end of the rib and this vortex will be increases with increasing $(p / H)$ as shown in (fig. $8 \mathrm{f}, g, h)$.

Heat transfer performed in term of Nu along the duct can be shown in the (fig. 9) for downstream and upstream ribs. The figure shows a part of the test section. Where $\mathrm{Nu}$ for the two types are decreases with increasing the distance $(x / H)$ due to the fluid will be hot toward the end of the duct also, Nux for downstream rib was higher than the upstream rib. The distribution of Nu along the duct for the two types at different Re can be seen in (fig.s 10 and 11 ), it is clear when increases Re lead to increasing heat transfer due to increasing the mixing fluid flow.

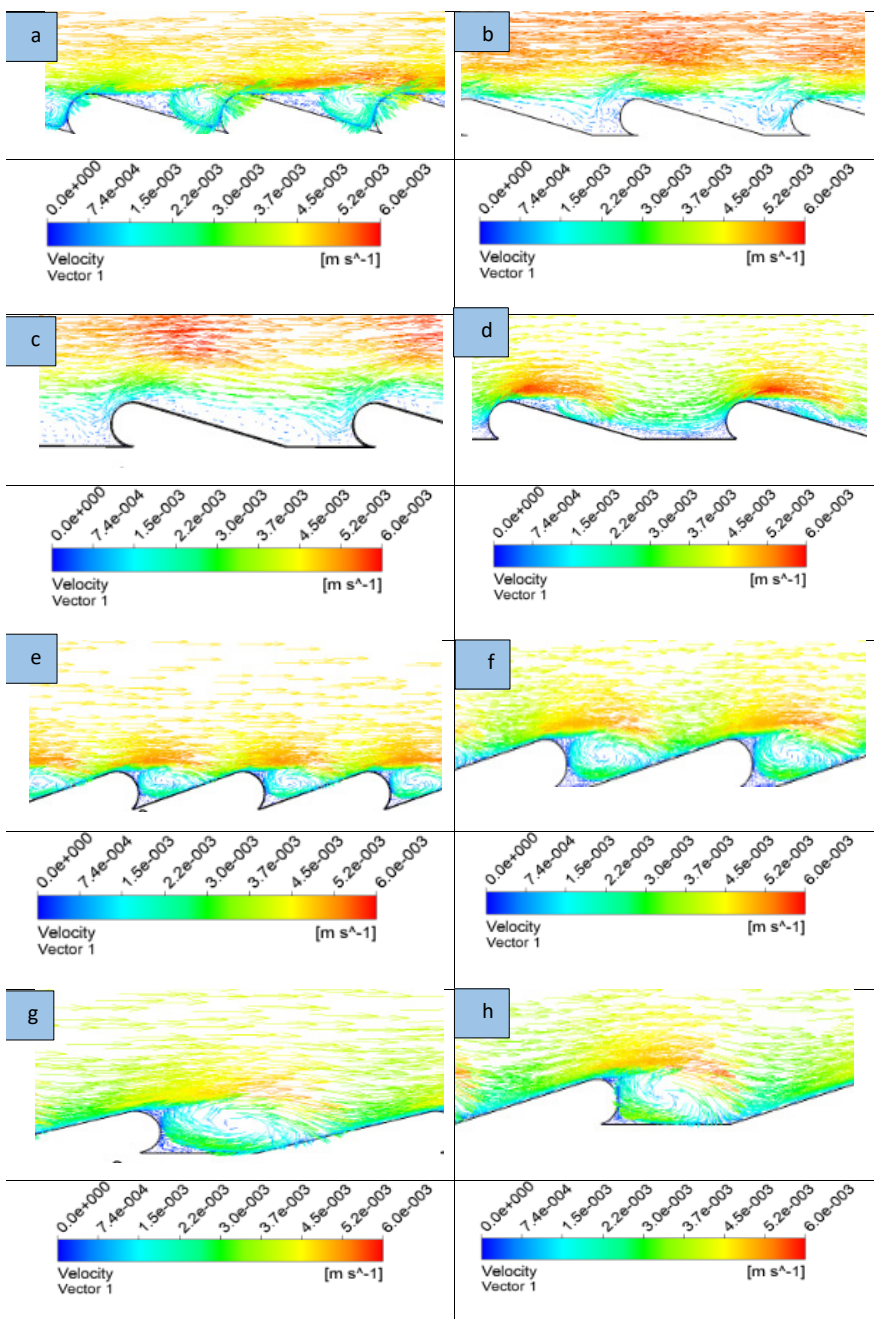

Figure 8: Velocity vector for downstream and upstream ribs 


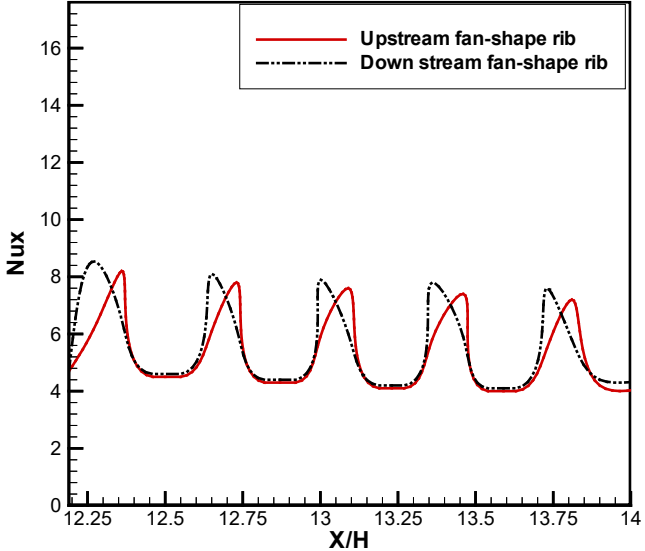

Figure 9: Local Nusselt number along the rib surface for two cases at $R e=800$

Figures (12 and 13) show the variation average Nuav with $\mathrm{Re}$ for downstream and upstream ribs respectively. It can be observing that Nu for duct with using rib is higher than the plain duct at any Re also, its shown that $\mathrm{Nu}$ increases with pitch ratio increasing, this due to good mixing of fluid flow and disappear of the vortices especially at downstream type.

Variation if friction factor (f) obtain for downstream and upstream types with $\mathrm{Re}$ under different pitch ratio are shown in fig. (14) and (15) respectively. From the figures can be observe that $(f)$ increases with increasing $(p / H)$ for upstream type due to growth the vortices but the opposite happens at downstream type where the vortices will be disappearing.

(Fig.16) shows the comparison of $\left(\mathrm{Nu} / \mathrm{Nu}_{\text {plain }}\right)$ between downstream and upstream with different pitch ratio $(\mathrm{p} / \mathrm{H})$ at $(R e=800)$, it is observed that heat transfer enhances for two case and the increasing of heat transfer or downstream was best and that lead to its possible to reduces the number of ribs at the same surface to decreasing friction factor.

\section{CONCLUSION}

In this study the effect of fan-shaped ribs on heat transfer and fluid flow parameters of laminar flow in a two-dimen-

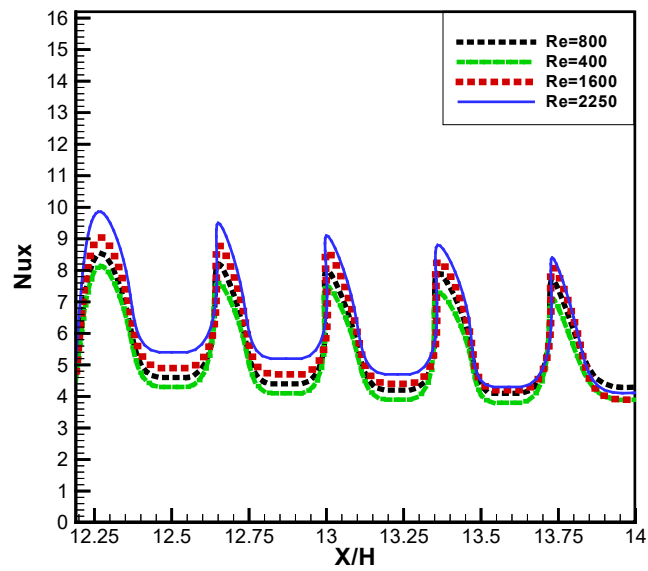

Figure 10: Local Nu along the test section at different Re for downstream case

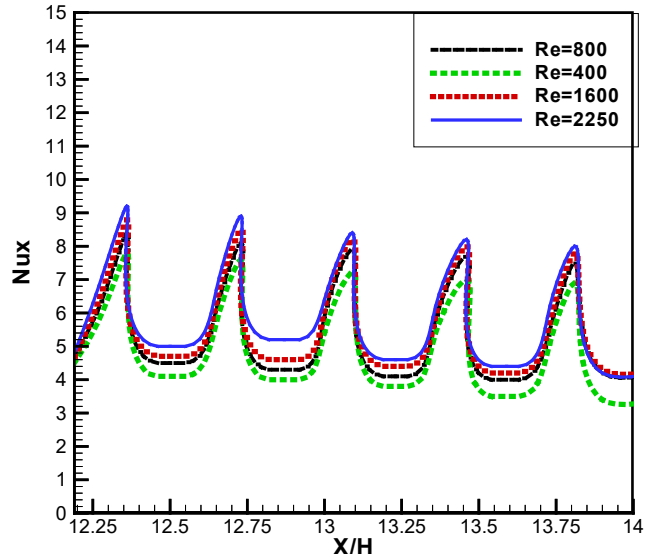

Figure 11: Local Nu along the test section at different Re for upstream case

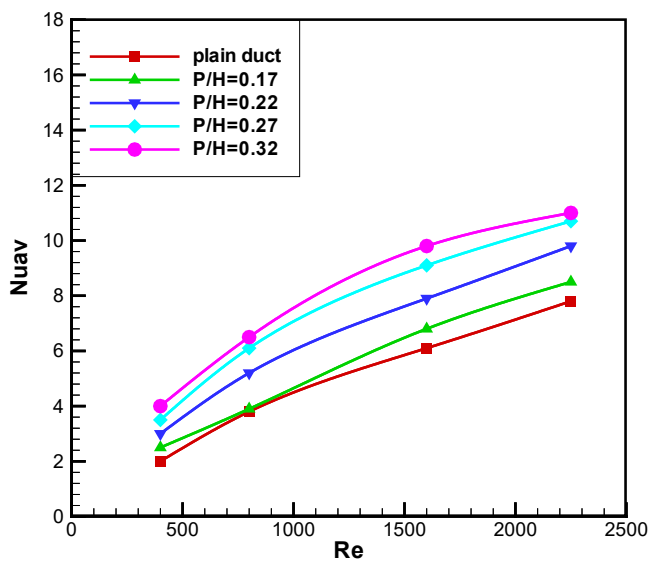

Figure 12: average $\mathrm{Nu}$ with $\mathrm{Re}$ at different (pH) for downstream case

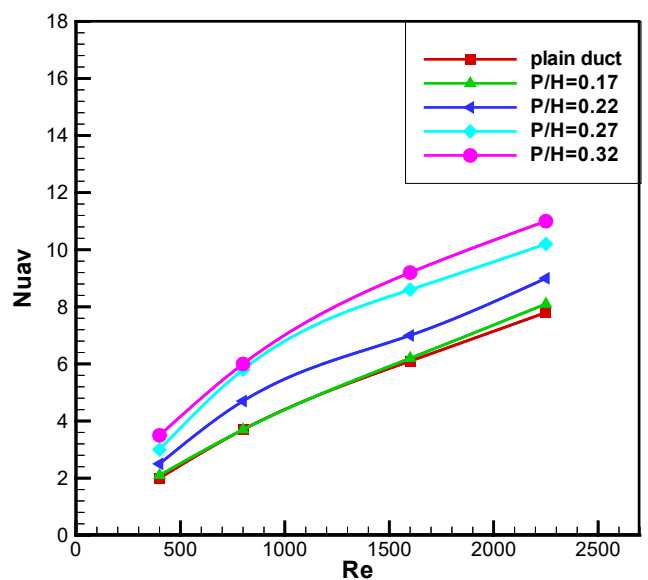

Figure 13: Average Nu with Re at different $(p H)$ for upstream case

sional rectangular duct was investigated. The FVM package was using ANSYS FLUENT 16.1 to simulate polling. In this study, the heat transfer and friction factor within the duct were examined in the Re range (400-2250). There are two cases of fan-shaped ribs, upstream and downstream of different pitches $(\mathrm{p} / \mathrm{H}=0.17,0.22,0.27,0.32)$. Through the studies in this paper, it can be shown that heat transfer was enhanced by the use of fan-shaped ribs and by an increase in Reynolds number. 


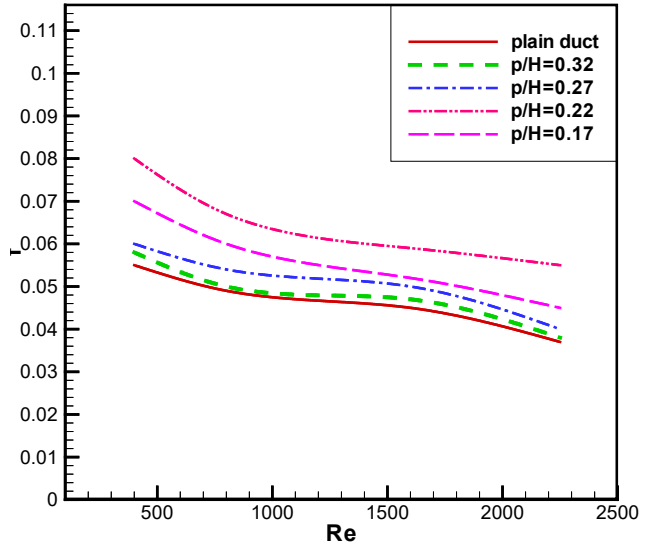

Figure 14: friction factor with $\mathrm{Re}$ at different $(\mathrm{pH})$ for downstream case

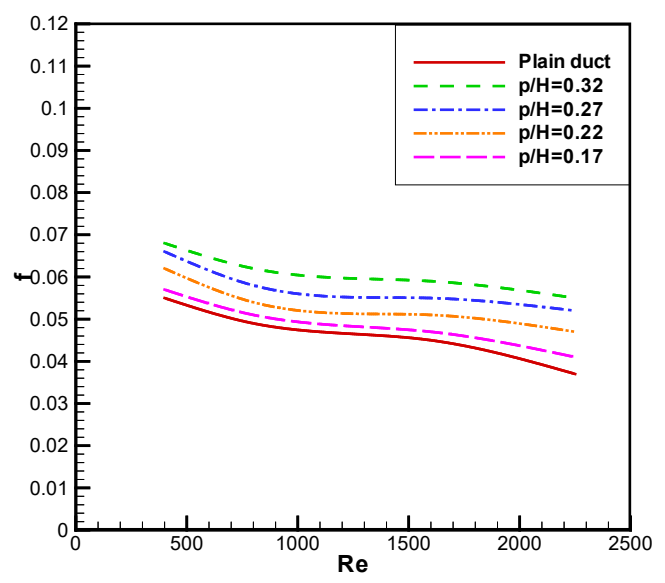

Figure 15: Friction factor with Re at different $(p H)$ for upstream case

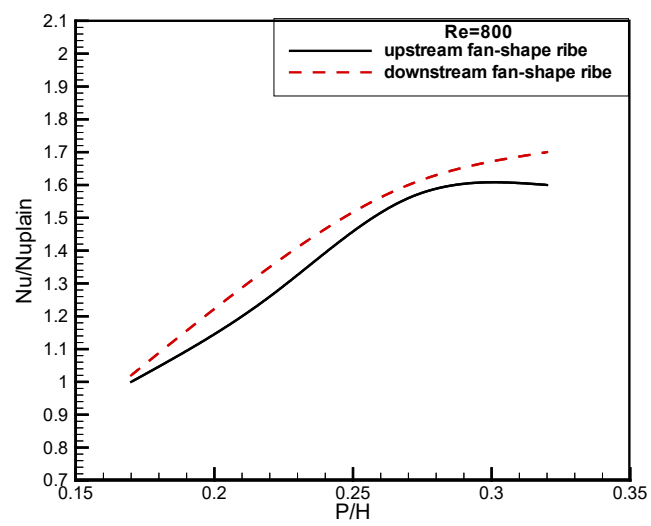

Figure 16: The comparison of $\left(\mathrm{Nu} / \mathrm{Nu}{ }_{\text {plain }}\right)$ between downstream and upstream with different pitch ratio at $(R e=800)$

Pitch ratio has a great influence on heat transfer and friction factor in two cases. Heat transfer improved by (12\%-29\%) when using fan shaped ribs and the maximum heat transfer occurred at $(\mathrm{p} / \mathrm{H}=0.32)$.

It has been found through the study that the vorteices that occur due to the presence of the ribs have a great effect on the heat transfer process, and that the changes made by changing the direction of the ribs or the dis- tance between them have a high effect on reducing the vorteiece and thus increasing the heat transfer.

Friction factor increases or decreases when using fanshaped ribs, as it increases with increasing pitch ratio to upstream condition and decreases with increasing pitch ratio to downstream condition. For all Reynolds numbers, the heat transfer for the downstream state was preferable to the upstream condition.

\section{REFERENCES}

1. A. Bhislek Kumar, M. Ayank Kumar Dwivedi, (2019). Determination of heat transfer coefficient and friction factors for turbulent flow through rectangular ducts with triangular ribs, International journal of scientific research and engineering development, 2 (3), 521-525.

2. Nabeel A. Ghyadh, (2015). Enhancement of heat transfer for air flow through a duct with various ribs, International journal of mechanical and production engineering research and development.5(5), 71-79.

3. Arjumand Rasool and Adnan Qayoun,(2018). Nemerical analysis of heat transfer and friction factor in two pass channels with variable rib shapes, International journal of heat and technology, 36(1),40-48.

4. A Poulemtafes- Boukadoum and A. Benzaoui (2014). CFD based analysis of heat transfer enhancement in solar air heater provided with transverse rectangular ribs Energy Procedia (50) 761 - 772.

5. Mr. Sandeep Kumar Karol, and Mr. Ishwar Singh, (2015). CFD based comparative analysis of different ribs with varying and same height in rectangular duct, International journal of science technology and engineering, 1 (10),6-15.

6. Riyadh S. Al-Turaihi, and Sarah Hasan Oleiwi, (2016). Heat transfer of two phases (water-air) in horizontal smooth and ribbed ducts, International journal of energy applications and technology, 3 (2) 41-49.

7. Pasaldi M. Kumar and Kattempudi R. Kumar, (2012). Enhancement of heat transfer of laminar flow in square ribbed duct with twisted tape, International journal of engineering science and technology,4(3), 3450-3456.

8. K. Ravi Kumar, M. Cheepu, B. Srinivas, D. Venkateswarly, G. P. Kumar, and A. Shiva, (2018). Analysis and comparison of solar air heater with various rib roughness using computational fluid dynamic (CFD), IOP Conf. series: Materials Science and Engineering 330, doi: 10.108811757-899x1330/1/012061.

9. Manjunath M. S., K. Vasudeve and N. Y. Sharma, (2017). Numerical analysis on the influence of novel semi-D shaped turbulator on heat transfer enhancement of solar air heater, 6th international conference on electronics, computer and manufacturing engineering, p.p. 245-249, ICECME, https:// doi-org/ 10.17758/EAP. U 0317108. 
10. Rajneesh Kumar, Anoop Kumar and Varun Goel. (2017) A parametric analysis of rectangular rib roughened triangular duct solar air heater using computational fluid dynamics. Solar Energy 157, 1095-1107.

11. Tuqa Abdulrazzaq, Hussein Togun, M. K. A. Ariffin, S. N. Kazi, N. M. Adam, and S. Masuri, (2013). Numerical simulation on heat transfer enhancement in channel by triangular ribs, International journal of mechanical and mechatronics engineering, 7(8), 1674-1678.

12. M. E. Taslim, and B. Ren, A combined numerical and experimental study on heat transfer in a cooling channel roughened with g: Ramped ribs, Proceedings of ISROMAC 2016 Honolulu, Hawaii, USA, April, 10-15.

13. A. Chaube, P. K. Sahoo and S. C. Solanki, (2006). Effect of roughness shape on heat transfer and flow friction characteristics of solar air heater with roughened absorber plate, Advanced computational methods in heat transfer (53), 41-51.

14. K. R. Chavan, N. D. Dhawale, D. N. Adling, K. P. Deshmukh, S. K. Kalukhe, and S. A. More, (2015). Experimental investigation of heat transfer analysis of ribbed duct for thermal per formal enhancement, International journal of science engineering and technology research (IJSETR), (4), 984-989.

15. Dr. Ekhlas M. Fayyadh, Dr. Moayed R. Hasan, and Sahira H. Ibrahim, (2016). The effect of variation longitudinal ribs height in spanwise direction on flow and heat transfer characteristics in a rectangular duct, Engineering and technology journal, 34, Part (A)(2),368-385.

16. Yadaba Mahanand, Abhijit Mahato, and Debanshu Shekhar Khamari, (2018). CFD analysis of semicircular rib roughened solar air heater, International journal of advanced mechanical engineering, 8 (1), 251-262.

17. Ds. Rawat, and Dr. A. R. Jaurkev, (2014). Enhancement of heat transfer using artificial roughness in solar air heater, International journal of engineering science invention, 3(6), 50-63.

18. Jasjeevan Singh, Harpuneet Singh, (2018). A Review on solar air heater using various roughness geometries on roughened duct to increase heat transfer coefficient and friction characteristics, International journal of advance research and innovation, 6(4),340-345.

19. Yilmas T., Erdinc, M. T., Fluid flow mixing for heat transfer enhancement in communicating converging and diverging channel, In. Ichmt Digital Library online, Begle House Inc.., 2014.
20. Menter F. R., (1994). Two equation Eddy-viscosity turbulence Model for engineering applications, American Institute of Aeronautics and Astronautics, 3(2), 283-300.

21. Rupesh J. Yadav, Sandeep Kore, V. N. Raibhole, and Prathamesh S. Joshi, (2015). Development of correlation for friction factor and heat transfer coefficient for square and Hex. duct with twisted tape insert in laminar flow, Procedia Engineering, 127, 256-257.

\section{NOMENCLATURE}

\begin{tabular}{|c|c|c|}
\hline Symbol & Description & Units \\
\hline $2-D$ & Two dimensions & \\
\hline B.C & Boundary condition & \\
\hline CFD & Computational fluid dynamic & \\
\hline $\mathrm{b}$ & Length of rib & M \\
\hline e & Height of rib & $\mathrm{m}$ \\
\hline $\mathrm{H}$ & Duct height & $\mathrm{m}$ \\
\hline $\mathrm{h}$ & Heat transfer coefficient & $\mathrm{Wm}^{-2} \mathrm{k}^{-1}$ \\
\hline $\mathrm{k}$ & thermal conductivity & $\mathrm{Wm}^{-1} \mathrm{k}^{-1}$ \\
\hline $\mathrm{L}_{1}$ & entrance length & $\mathrm{m}$ \\
\hline $\mathrm{L}_{2}$ & Test section & $\mathrm{m}$ \\
\hline $\mathrm{L}_{3}$ & Exit section & $\mathrm{m}$ \\
\hline $\mathrm{Nu}$ & Nusselt number & \\
\hline $\mathrm{Pr}$ & Prandtl number & \\
\hline$P$ & Pressure & $\mathrm{Pa}$ \\
\hline $\mathrm{p}$ & Pitch & $\mathrm{m}$ \\
\hline $\mathrm{T}$ & Temperature & $\mathrm{K}$ \\
\hline $\mathrm{u}$ & Horizontal velocity & $\mathrm{m} / \mathrm{sec}$ \\
\hline $\mathrm{v}$ & Vertical velocity & $\mathrm{m} / \mathrm{sec}$ \\
\hline $\mathrm{Re}$ & Reynolds number & \\
\hline Dh & Hydraulic diameter & $\mathrm{m}$ \\
\hline
\end{tabular}

